\section{Introduction to the Synthesis and Purification of Oligonucleotides}

Avery's realization that DNA carries the genetic information lead chemists on a 40-year search that has culminated in efficient, automated oligonucleotide synthesis on solidphase supports. Modern nucleic acid synthesizers utilize phosphite triester chemistries that employ stable phosphoramidite monomers to build the growing polymer (see UNIT 3.3). These robust reactions allow both chemists and molecular biologists to easily generate specific ribo- and deoxyribo oligonucleotides with a variety of labels, modified linkages, and nonstandard bases attached throughout the chain. The synthesis of short (less than 40-nucleotide) probes and primers requires no more special expertise than the ability to read the synthesizer operator's manual, and longer oligonucleotides (up to 150 nucleotides) can be synthesized with greater care.

The introductory section of this appendix provides strategies for the maximization of synthetic yield, the generation of sequences containing site-specific modifications, and the isolation of synthetic oligonucleotides. Protocols describe monitoring the progress of synthesis via the trityl assay (see Basic Protocol 1 and Support Protocol) and methods for deprotection of DNA (see Basic Protocol 2) and RNA (see Basic Protocol 3) oligonucleotides.

This appendix augments the detailed instructions provided by the manufacturers of oligonucleotide synthesizers. A functional understanding of the synthesis chemistries, coupled to insights on the mechanical operation of the synthesizer, will allow the user to minimize input time and maximize oligonucleotide output.

\section{INTRODUCTION TO CHEMICAL NUCLEIC ACID SYNTHESIS}

Many hydroxyl and amine moieties make nucleic acids very animated molecules with rich chemistries of their own that can interfere with the phosphite triester reactions used to couple the nucleotide monomers; therefore, protection strategies are necessary in chemical synthesis to mask the functional groups on the monomers so that the only significant reaction is the desired $3^{\prime}$ to $5^{\prime}$ sequential condensation of monomers to the growing oligonucleotide. These synthetic protecting groups must be chosen so that they can be removed easily to expose the natural nucleotides.

The fully protected monomers for nucleic acid synthesis are generally called phosphoramidites (Fig. A.3C.1; also see recipe in Reagents and Solutions). In traditional protection schemes the nucleophilic amino moieties on the bases are protected (see UNIT 2.1) with either isobutyryl (N-2 of guanine) or benzoyl (N-6 of adenine and N-4 of cytidine) groups, both of which can be removed at the completion of synthesis by ammoniolysis. However, recent advances have lead to the widespread use of phenoxyacetyl (PAC) protection of adenosine, dimethylformadine $(\mathrm{DmF})$ protection of guanosine, and acyl protection of cytosine to yield oligonucleotides that can be deprotected rapidly under very mild conditions (Reddy et al., 1994). The 5' primary hydroxyl of the ribose sugar is protected (see UNIT 2.3) with a dimethoxytrityl (DMT) ether moiety which is removed by mild protic acids at the beginning of each coupling cycle. The efficiency of synthesis at each coupling cycle can be monitored by detecting the release of the chromophore trityl cation. To synthesize nucleic acids with the natural phosphodiester backbone, the $3^{\prime}$ secondary hydroxyl function of the ribose sugar is derivatized with a highly reactive phosphitylating agent. The phosphate oxygen on this moiety is usually masked by $\beta$-cyanoethoxy and diisopropylamine protecting groups. By insulating the phosphate oxygen with alternative groups, modified phosphate backbones may be accessed. Finally,

Contributed by Andrew Ellington and Jack D. Pollard, Jr. Current Protocols in Nucleic Acid Chemistry (2000) A.3C.1-A.3C.22

Copyright $(2000$ by John Wiley \& Sons, Inc.
Commonly Used Techniques 
for ribonucleic acids the secondary $2^{\prime}$ hydroxyl of the ribose is shielded throughout the chemical synthesis by the tert-butyldimethylsilyl group (UNIT 2.2).

The convenience of using these protecting groups for automated nucleic acid synthesis is that they yield nearly lesion-free natural nucleic acids with high efficiency through simple hydrolysis, nucleophilic displacement, and redox chemistries. In a standard synthesis cycle, the nucleotide chain grows from an initial protected nucleoside derivatized via its terminal 3' hydroxyl to a solid support (see UNIT 3.1). Reagents and solvents are pumped through the support to induce the consecutive removal and addition of sugar protecting groups in order to isolate the reactivity of a specific chemical moiety on the monomer and effect its stepwise addition to the growing oligonucleotide chain. This design eliminates the need to purify synthetic intermediates or unreacted reagents, because they

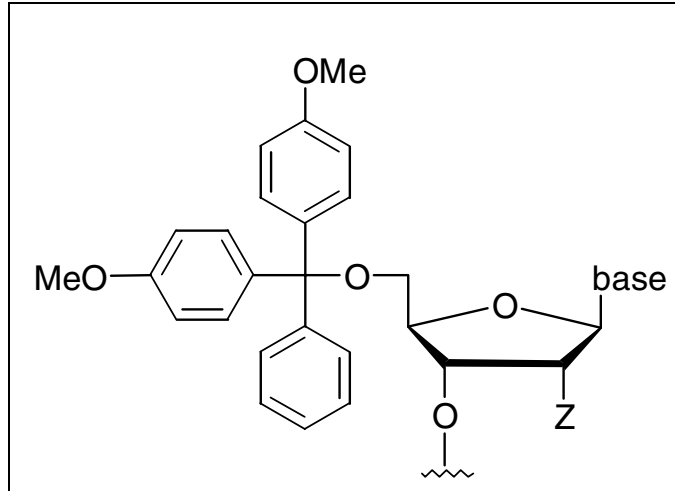

5 ' dimethoxytrityl protection

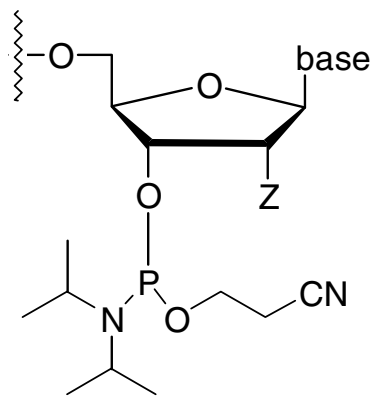

$\beta$-cyanoethyl phosphorus protection<smiles>CC(C)C(=O)Cc1nc2c(ncn2C(C)C)c(=O)[nH]1</smiles>

isobutyrylguanine protection

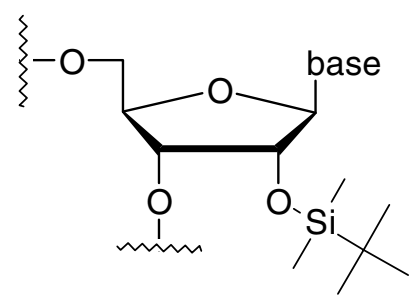

$2^{\prime} t$-butyldimethylsilyl protection (Z)<smiles>Cn1ccc(NC(=O)c2ccccc2)nc1=O</smiles>

benzoylcytidine protection<smiles>Cn1cnc2c(NC(=O)c3ccccc3)ncnc21</smiles>

benzoyladenine protection
Introduction to the Synthesis and Purification of Oligonucleotides
Figure A.3C.1 Structure of nucleoside phosphoramidite units, showing traditional protection groups for 2' hydroxyl, phosphate, and base moieties. For DNA, $\mathrm{Z}=\mathrm{H}$ (no protection required); for RNA, Z = O-protection group. 
are simply rinsed off the column at the end of each chemical step. Assembly of the protected oligonucleotide chain is carried out in four chemical steps: deblocking, activation/coupling, oxidation, and capping (Fig. A.3C.2). Cleavage and deprotection then reveal the single-stranded nucleic acid.

\section{Deblocking}

The synthesis cycle begins with the removal of the acid-labile DMT ether from the $5^{\prime}$ hydroxyl of the $3^{\prime}$ terminal nucleoside. This is usually accomplished by using dichloroacetic acid (DCA) in dichloromethane. The resulting trityl cation chromophore can be quantitated to determine coupling efficiency (see Basic Protocol 1). After deblocking, the $5^{\prime}$ hydroxyl is the only reactive nucleophile capable of participating in the subsequent coupling step. Since the nitrogenous bases of the growing DNA chain are susceptible to acid-catalyzed depurination, the deblocking step is short, and an acetonitrile rinse thoroughly removes the deblocking agent from the support. Also, coupling efficiency and accuracy are increased by this wash, since premature detritylation of the incoming phosphoramidite monomer is prevented.

\section{Activation/Coupling}

Following deblocking of the $5^{\prime}$ hydroxyl group, the next protected phosphoramidite is delivered to the reaction column along with the weakly acidic activator tetrazole $\left(\mathrm{pK}_{\mathrm{a}}=\right.$ 4.8). Nucleophilic attack of the previously freed $5^{\prime}$ hydroxyl upon the incoming monomer elongates the nucleic acid chain. Because this protonated phosphoramidite is so reactive, the coupling reaction is usually complete within $30 \mathrm{sec}$. A molar excess of tetrazole over the phosphoramidite ensures complete activation, and a molar excess of phosphoramidite over free $5^{\prime}$ hydroxyls of the growing chain promotes efficient coupling. To optimize the coupling efficiency, the amounts of reagents injected and the coupling time can be varied (see Synthesizing Long Oligonucleotides).

\section{Capping}

In spite of these efficiency measures, a small percentage of the support-bound nucleoside's $5^{\prime}$ hydroxyls do not couple to the incoming activated monomer. They must be rendered inactive to minimize deletion products and simplify the purification process. Usually, acetic anhydride and $N$-methylimidazole dissolved in pyridine and tetrahydrofuran (THF) act to create an acylating agent that "caps" the unextended 5' hydroxyls. The $5^{\prime}$ acetyl ester cap is unreactive in all subsequent cycles and is removed during the final ammonia deprotection step. Additional acetonitrile washing subsequent to capping can increase synthetic yield. After coupling and capping, the internucleotide linkage is a trivalent phosphite triester that is extremely unstable and must be oxidized to a phosphotriester, which will ultimately yield natural DNA.

\section{Oxidation}

In the last step of the cycle, the unstable phosphite triester linkages are oxidized to a more stable phosphotriester by $0.02 \mathrm{M}$ iodine dissolved in water/pyridine/THF (see recipe for oxidizer). An iodine-pyridine adduct forms to the phosphite triester and is subsequently displaced by water to yield phosphorus oxidized to the pentavalent state. Pyridine also neutralizes the hydrogen iodide byproduct. Because the oxidizer contains water, the support is rinsed several times with acetonitrile following this reaction. One cycle of monomer addition is then complete, and another cycle begins with the removal of the $5^{\prime}$ DMT from the previously added monomer.

\section{Cleavage/Deprotection}

At the end of the synthesis, the final trityl can either be removed with a final acid wash ("trityl-off"), or be left on for purification purposes ("trityl-on"). The oligonucleotide

Commonly Used Techniques 


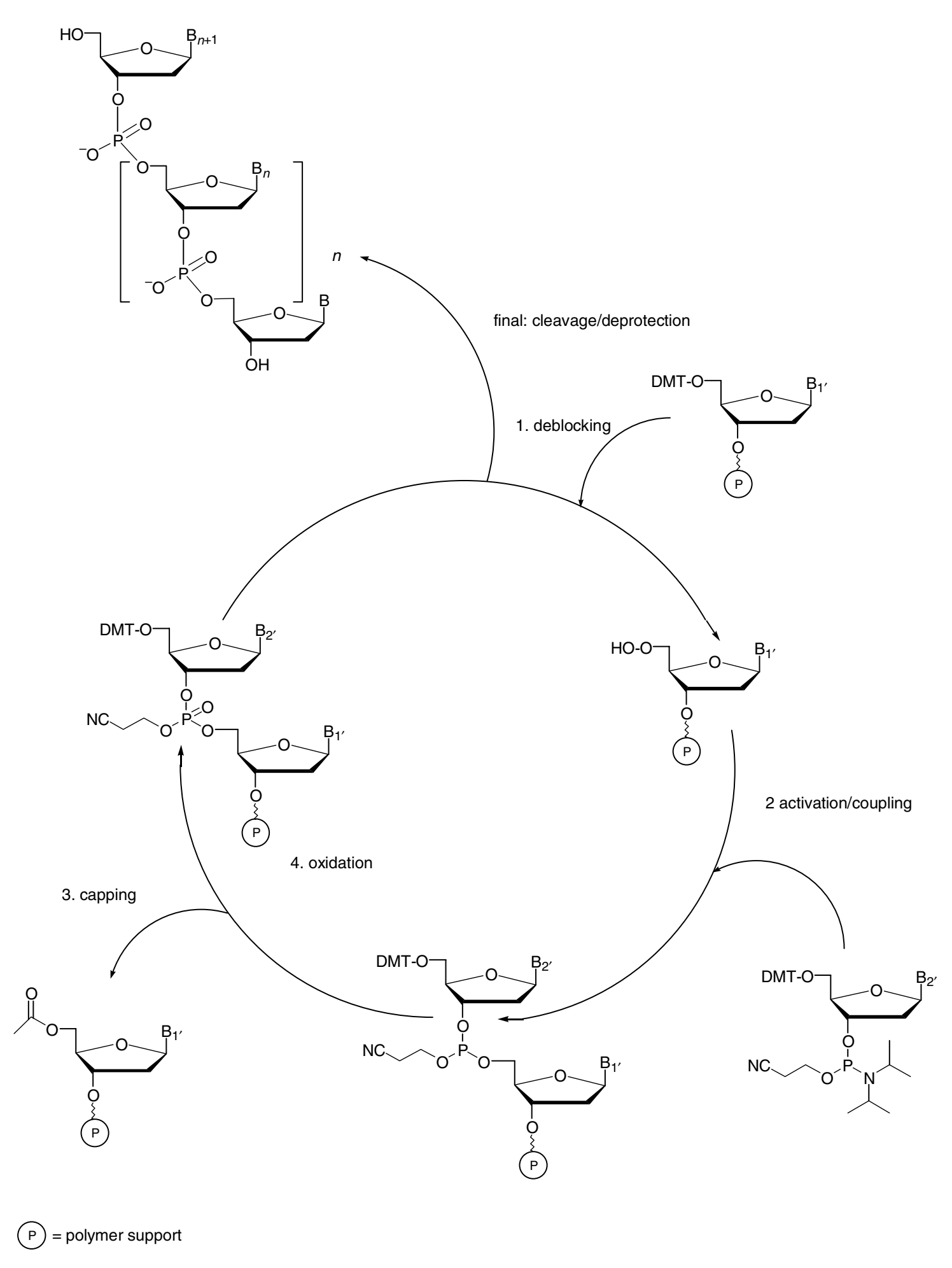

Figure A.3C.2 Steps in the assembly of the protected oligonucleotide chain.

Introduction to the Synthesis and

Purification of

Oligonucleotides 
itself is removed from the support with concentrated ammonium hydroxide. Additionally, this treatment deprotects the phosphorus by $\beta$-elimination of the cyanoethyl group and removes the protecting groups from the heterocyclic bases to yield a single-stranded nucleic acid.

\section{RNA Synthesis}

RNA chemical synthesis is identical to that used for DNA except for the need for an additional protecting group at the $2^{\prime}$ hydroxyl of ribose. This position is usually protected with tert-butyldimethyl silyl groups, which are stable throughout the synthesis (Fig. A.3C.1). They are removed at the final deprotection step by addition of a basic fluoride ion such as tetrabutylammonium fluoride (TBAF). The remaining positions on both the sugar and the bases are protected in the same fashion as for DNA. By adjusting several parameters in the DNA synthesis protocol-including the coupling times, monomer delivery rate, frequency of washing steps, and types of capping reagents-stepwise coupling efficiencies of up to $99 \%$ can be obtained (Wincott et al., 1995; G. Glick, pers. comm.). However, for the casual user this yield represents the exception rather than the norm, and only shorter oligoribonucleotides ( $<20$ bases) should initially be attempted.

\section{STRATEGIES FOR NUCLEIC ACID SYNTHESIS}

\section{A Checklist for Nucleic Acid Synthesis}

Consistency and planning are the keys to reliable nucleic acid synthesis. Organizing these repetitive tasks into a standard operating protocol will streamline efficiency and produce better-quality oligonucleotides.

1. Plan syntheses to optimize machine use. Oligonucleotides of similar size should be combined in parallel runs, since synthesizing many short oligonucleotides followed by a longer one is faster than mixing the sets on dual-column synthesizers.

2. Determine the total number of bases to be incorporated. Be sure there are enough of the required reagents and phosphoramidites available for the entire synthesis. Consult the synthesizer manual for the amount of reagent needed for each coupling (also see Troubleshooting). Remember that different synthetic scales (e.g., $0.25 \mu \mathrm{mol}$ versus $1 \mu \mathrm{mol})$ require different amounts of reagents. Syntheses should be planned so that a phosphoramidite is almost completely exhausted. Also, phosphoramidites that have been dissolved for $>2$ weeks should be replaced.

3. Consider special programming requirements. Many synthesis options pertaining to the scale of the synthesis, backbone composition, and presence of protecting groups can be modified. Create a log for users to fill out detailing exact synthesis requirements, and check the log prior to synthesis. A computerized log book is especially useful and allows for an organized oligonucleotide nomenclature (e.g., R20.17 may refer to Rebecca's 20-mer, the 17th 20-mer made on the system).

4. If the synthesizer lacks a trityl monitor, set up a fraction collector to monitor the chromophoric trityl cation release (see Basic Protocol 1).

5. Initialize the system. If previous oligonucleotides were cleaved from their supports automatically, rinse the columns for $30 \mathrm{sec}$ with acetonitrile to remove any remaining traces of ammonium hydroxide. If the machine has been at rest for $>6 \mathrm{hr}$, immediately before starting the synthesis (and following the addition of fresh reagents) remove any stale reagents or moisture from the lines by priming them. This will maximize 
the first coupling step's efficiency. Check the reagent and phosphoramidite flow rates to ensure that reagents are being properly delivered.

6. Start the synthesis. Confirm that the flow through the lines and columns is not obstructed and monitor the first few trityl releases. An abortive synthesis of a 60-mer sequence wastes much more material than a failed 3-mer run.

\section{Synthesizing Long Oligonucleotides ( $\geq 100$ Bases)}

Modern synthesizers can routinely produce sequences of 150 or more nucleotides in usable amounts $(\geq 10 \mu \mathrm{g})$. Several strategies can be employed to enhance the yield from syntheses longer than 100 bases (UNIT 3.3).

1. Exclude water from the system (the importance of this cannot be overstated; see Critical Parameters). Replace reagents on the machine with fresh ones before all long syntheses. This is particularly important for the phosphoramidites and especially for guanosine phosphoramidite, since it decomposes more quickly than the other two protected bases (Zon et al., 1985).

2. Monitor trityl releases for shorter runs prior to attempting the synthesis of long oligonucleotides to limit wastage of expensive reagents. In general, if the stepwise efficiency of synthesis is $<99 \%$, alter parameters to increase the efficiency on shorter sequences before attempting to synthesize a long oligonucleotide (see Basic Proto$\operatorname{col} 1)$.

3. Use dichloroacetic acid (DCA) rather than trichloroacetic acid (TCA) for deblocking if the synthesizer is compatible with this reagent. Depurination (cleavage of the glycosidic bond) under acidic conditions is a prominent side reaction that ultimately limits DNA synthesis. DCA tends to show much better synthetic yields than trichloroacetic acid, especially for longer oligonucleotides (R. Pon, pers. comm.). Use a $2 \%$ (v/v) DCA/1,2-dichloroethane mixture.

4. Modify the synthesis protocol to increase the coupling time of the phosphoramidite. Also, additional methylene chloride wash steps included prior and subsequent to deblocking, along with increased acetonitrile washing subsequent to capping, lead to increased yields (G. Glick, pers. comm.).

5. Increase the phosphoramidite concentration to enhance the coupling efficiency-e.g., use a concentration of $50 \mathrm{mg} / \mathrm{ml}$ (double the normal concentration; 20-fold molar excess over the synthetic polynucleotide chain) for longer sequences.

6. Using a support matrix such as control pore glass (CPG; UNIT 3.1) with a loading capacity of $<40 \mu \mathrm{mol} / \mathrm{g}$ can greatly increase yields of long oligonucleotide. Furthermore, the pore size of the support should be $1000 \AA$ for $>100$-mers and $2000 \AA$ for 200 -mers (Gait, 1984) to alleviate molecular crowding and steric effects. For a typical $1-\mu \mathrm{mol}-$ scale synthesis of a 150 -mer, $20 \mathrm{mg}$ of a support with a loading capacity of $5 \mathrm{mmol} / \mathrm{g}$ is used.

If it is too difficult to synthesize the desired sequence in a reasonable yield, or if oligomers $>150$ bases are desired, the nucleic acids may be made in segments and ligated (Bartel and Szostak, 1993) following PCR with a proofreading polymerase such as Pfu or Tth. Note, however, that since PCR is usually a mutagenic procedure, any products generated should be checked by sequencing. Also, very long synthetic oligonucleotides (300 to 600 bases) have also been synthesized directly, and in spite of incredibly low yields, rare

Introduction to the Synthesis and Purification of Oligonucleotides full-length products have been successfully amplified by PCR (Ciccarelli et al., 1991). 
Finally, mutually primed synthesis (e.g., CPMB UNIT 8.2) can also be a suitable option for oligomers $>150$ bases.

\section{Synthesizing RNA}

Since many structural and mechanistic studies are underway concerning catalytic RNAs, the catalog of commercially available modified RNA monomers has recently bloomed (Table A.3C.1). RNA chemical synthesis (see UNIT 3.5) has become as routine as that of DNA (see UNIT 3.3) and typically uses identical $5^{\prime}$-dimethoxytrityl- $\beta$-cyanoethyl-protected phosphoramidites except for an additional protecting group on the $2^{\prime}$ hydroxyl (see UNIT 2.2). tert-Butyldimethylsilyl protection of the 2 '-hydroxyl group is the basis of most commercially available RNA phosphoramidites, since the silyl group is stable to both acid and base and can be removed with fluoride ion. Recently, however, RNA monomers with 2'-acetal groups (FPMP, CEE; also see UNIT 2.2) have appeared; these have the advantage of being conveniently removed at $\mathrm{pH} 2$ just prior to use of the particular sample of RNA. Strategies for ribophosphoramidite protection are an active area of research, and recent work with $5^{\prime}$-silyl ethers in conjunction with $2^{\prime}$-orthoester protection has proven particularly interesting (S. Scaaringe, pers. comm.). For the casual user of RNA, it is often easier just to purchase small quantities of the required sequence from a ribo-oligonucleotide synthesis company such as Baron Consulting, Dharmacon Research, Genosys, or Peninsula Labs (see SUPPLIERS APPENDIX).

Isomeric purity of the phosphoramidites is often variable because of the difficulties inherent in distinguishing between the vicinal hydroxyls of ribose. If a homogeneous population of $5^{\prime}$-to-3'-linked oligoribonucleotides is required (as in most cases), then a thin-layer chromatography (TLC) or ${ }^{31} \mathrm{P}$ nuclear magnetic resonance (NMR) analysis of the starting phosphoramidites should be performed to establish their isomeric composition. While phosphorus NMR facilities are not generally available to molecular biologists, TLC is both inexpensive and straightforward. Recommended solvent systems to separate the $2^{\prime}$ and $3^{\prime}$ ribonucleotide phosphoramidites are 1:1 ether/chloroform, or $40: 58: 2$ or 50:46:4 dichloromethane/hexane/triethylamine (Usman et al., 1987).

With a few slight modifications, the procedures and precautions described for DNA synthesis chemistry apply to RNA as well (UNIT 3.5). Since stepwise coupling efficiency is lower than that of DNA, even greater care should be taken to exclude water completely from the closed system. Because the $2^{\prime}$ hydroxyl is often protected with sterically hindering protecting groups, reaction times for RNA reagents tend to be longer, and adjustments should be made to phosphoramidite concentrations and coupling times, as detailed below. As is true for all RNA work, equipment and reagents that will contact unprotected oligonucleotides should be RNase free to avoid degradation of the synthesized material.

Depending on the synthesizer and coupling program used, RNA phosphoramidites are suspended in dry acetonitrile at a concentration of 0.1 to $1.0 \mathrm{M}$ with a 6- to 10 -fold excess of reagents delivered per $300 \mathrm{sec}$ coupling. $S$-ethyltetrazole and DCI have also been found to be a more effective activators than the traditional tetrazole (Sproat et al., 1995; Vargeese et al, 1998). Also, additional methylene chloride washing steps included prior to and subsequent to deblocking, along with increased acetonitrile washing subsequent to capping, lead to increased yields (G. Glick, pers. comm.). 
Table A.3C.1 Suppliers of Unnatural and Modified Phosphoramidites

\begin{tabular}{|c|c|}
\hline Phosphoramidite type $^{a}$ & Suppliers $^{b}$ \\
\hline Fast/mild deprotecting DNA and RNA monomers & $\begin{array}{l}\text { Cruachem } \\
\text { Glen Research } \\
\text { Perkin-Elmer } \\
\text { BioGenex } \\
\text { ChemGenes } \\
\text { PerSeptive BioSystems } \\
\text { Sigma }\end{array}$ \\
\hline Modified DNA monomers & $\begin{array}{l}\text { Appligene } \\
\text { Interactiva BioTechnologie } \\
\text { Glen } \\
\text { ChemGenes } \\
\text { Cruachem } \\
\text { Sigma } \\
\text { Perkin-Elmer } \\
\text { Solid Phase Science }\end{array}$ \\
\hline Convertible deoxynucleosides & Glen Research \\
\hline Radiolabeled deoxyphosphoramidites & Cambridge Isotopes \\
\hline Modified RNA monomers & $\begin{array}{l}\text { ChemGenes } \\
\text { BioGenex } \\
\text { Boehringer Mannheim } \\
\text { Glen Research } \\
\text { Dalton } \\
\text { Perkin-Elmer } \\
\text { Sigma } \\
\text { Cruachem }\end{array}$ \\
\hline Labeling monomers & $\begin{array}{l}\text { Clontech } \\
\text { Cruachem } \\
\text { Glen Research } \\
\text { Solid Phase Science } \\
\text { Perkin-Elmer } \\
\text { BioGenex } \\
\text { Sigma } \\
\text { ChemGenes } \\
\text { Boehringer Mannheim } \\
\text { ABI Biotechnology } \\
\text { PerSeptive BioSystems } \\
\text { Genosys }\end{array}$ \\
\hline $\begin{array}{l}\text { Uniquely structured oligonucleotide branched } \\
\text { or cyclic }\end{array}$ & $\begin{array}{l}\text { Clontech } \\
\text { Glen Research }\end{array}$ \\
\hline Non-enzymatically extended $3^{\prime}$ ends & Glen Research \\
\hline Alternative backbones & $\begin{array}{l}\text { Glen Research } \\
\text { ChemGenes }\end{array}$ \\
\hline
\end{tabular}

Introduction to the Synthesis and Purification of Oligonucleotides

${ }^{a}$ All phosphoramidites are 5' DMT and 3' cyanoethyl protected (CED) unless otherwise noted

${ }^{b}$ See Suppliers Appendix for supplier contact information. 
Incorporation of Modified Nucleosides

Chemical nucleic acid synthesis allows for the incorporation of unnatural or modified bases, as well as a variety of labeling moieties, into an oligonucleotide (see Chapter 4). This can be extremely useful for testing models of structural interactions between enzymes and nucleic acids, selecting labeled molecules from a population of unlabeled ones, or gaining insights into the parameters that govern nucleic acid structure and chemistry. Modified backbone chemistries such as phosphorothioates, phosphoroamidates, and phosphotriesters are also readily available. In general, the bases themselves can be obtained commercially and are handled like any other phosphoramidite; however, consult the company that supplies the analog about necessary modifications to programs or reagents (see Table A.3C.1). Typically, the only adjustment needed is to dissolve the modified base at a somewhat higher concentration than normal to overcome problems associated with reactivity. Most of the methods used to increase the yield of long and ribo-oligonucleotides may be applied to the synthesis of modified nucleic acids.

When synthesizing modified oligonucleotides, compatibilities of the chemistries, placement of modifications relative to other chemical groups, and $5^{\prime}$ to $3^{\prime}$ directionality are all factors to consider. Generally when an oligonucleotide is end-labeled/modified, a long flexible tether is added to allow greater accessibility. Stretches of four deoxythymines are often used for this purpose. Also, adding deoxythymines $5^{\prime}$ to the label (5'-TTTT-label-3') can aid in separating labeled molecules from unlabeled ones by size. Note that some tagging phosphoramidites allow for the enzymatic extension or kinasing of the modified oligonucleotide, while others do not. Finally, oligonucleotides may also be synthesized directly on solid glass supports (Cohen et al., 1997).

Terminal transferase can be used as an alternative means of incorporating modified bases at the $3^{\prime}$ end of an oligonucleotide (Ratliff, 1982; e.g., CPMB UNIT 3.6). This enzyme is tolerant of a variety of substrates, and has been used to add deoxynucleotide triphosphates derivatized at virtually every position ( $\mathrm{C}-8$ on adenine, any of the amino groups, $\mathrm{C}-5$ on cytosine, O-6 on guanosine) to DNA. It also functions, though less well, with RNA bases. It can use any DNA oligonucleotide that is at least 2 bases long $[\mathrm{d}(\mathrm{pXpX})]$ and contains a free $3^{\prime}$ hydroxyl as a primer. A potential problem in preparing homogeneous polynucleotides using terminal transferase is that a statistically random number of bases is added to the $3^{\prime}$ end of the template (with the exception of molecules such as cordycepin, which act as chain terminators due to the absence of $3^{\prime}$ hydroxyl). If a single species is desired, it can be gel purified. Polynucleotide phosphorylase may also be used to incorporate modified bases at the $3^{\prime}$ end (Gillam and Smith, 1980).

A more controlled means of introducing modified nucleotides relies on T4 RNA ligase and substrates of the form $\mathrm{A}\left(5^{\prime}\right) \mathrm{ppX}$ (where $\mathrm{X}$ can be virtually any molecule, including for example ribose or amino acids, in a pyrophosphate linkage with adenosine; Uhlenbeck and Gumport, 1982). The minimal template for reactions of this form is a trinucleoside containing a free $3^{\prime}$ hydroxyl. RNA reacts much better than DNA, and single-strand molecules act as better templates than double-stranded ones. Since $3^{\prime}$ hydroxyl groups are required, substrates of the form $\mathrm{A}\left(5^{\prime}\right) \mathrm{ppXp}$ will undergo only a single round of addition, unlike the similar reaction with terminal transferase. In some cases, the compound $\mathrm{A}\left(5^{\prime}\right) \mathrm{ppXp}$ can be generated directly by RNA ligase from $\mathrm{pXp}$ and ATP, although the substrate requirements for the $\mathrm{X}$ moiety are much more strict than in the ligation reaction. Thus, while virtually any dinucleotide of the form $\mathrm{A}\left(5^{\prime}\right) \mathrm{ppX}$ can be added to an oligonucleotide, only a few compounds (primarily sterically "small" derivatives of natural bases) can be used by the enzyme to form $\mathrm{A}\left(5^{\prime}\right) \mathrm{ppXp}$ from $\mathrm{pXp}$. 
T4 RNA ligase can catalyze the ligation of single-stranded oligonucleotides in the presence of ATP and various analogs (Kinoshita et al., 1997). Templates prepared by terminal transferase or by T4 RNA ligase that contain modified nucleotides (or other adducts) at their $3^{\prime}$ termini may be able to act as substrates in this reaction. This would allow modified nucleotides to be introduced into the middle of a longer chain. However, the substrate specificity of the enzyme for the $3^{\prime}$ hydroxyl donor is highly substrate dependent and will have to be determined empirically.

\section{Synthesizing Degenerate Oligonucleotides}

Current combinatorial and "irrational" nucleic acid design methodologies focus on the ability to create large pools of random sequences from which useful sequences may be culled (Szostak, 1992; also see Chapter 9). In addition, random mutagenesis using degenerate oligonucleotides allows for the exploration of "sequence space" surrounding a given protein or RNA structure. Sequences can be produced that give a completely random distribution of nucleotides at a given position or, alternatively, the sequence can be biased or "doped" toward a particular base with only a low level of randomization.

Most synthesizers can be programmed for in-line degenerate mixing of bases, which is useful if only a few positions are to be randomized. A potential problem with this method is that, if mixing is incomplete, the sequence will be skewed toward whichever phosphoramidite enters the column first, since the reaction of the activated phosphoramidite with the free $5^{\prime}$ hydroxyl is extremely fast. Therefore, while in-line mixing will generate all base substitutions at a given position, the distribution of these substitutions may not be uniform. If a statistically random distribution of nucleotides is required or if long stretches of random sequence are to be made, it is better to manually mix the phosphoramidites together and use this mixture for the degenerate position. A true random distribution may be obtained by mixing A, C, G, and T phosphoramidites in a 3:3:2:2 molar ratio to compensate for the faster coupling times of $\mathrm{G}$ and $\mathrm{T}$ phosphoramidites (D. Bartel, pers. comm.). On synthesizers where phosphoramidites are loaded without detaching the bottle, the mixing generated by sequential loading of each phosphoramidite into the extra bottle is sufficient to generate randomized sequences.

Oliphant and Struhl (Oliphant et al., 1986; Oliphant, 1989) have constructed degenerate oligonucleotides using mixed phosphoramidites, but have modified the synthesis protocol by deleting the capping step during the random additions. This increases the overall yield of long oligonucleotides, since sequences that fail to elongate are not terminated, and the size of the final product is more heterogeneous. This method is particularly useful if deletions, as well as randomized bases, in a given sequence are required.

Hermes et al. (unpub. observ.) have developed a detailed protocol for producing statistically mutated oligonucleotides. This method employs in-line mixing between pure phosphoramidite contained in separate bottles and equimolar mixtures of the four bases contained in an additional bottle. The obvious advantage of such a method is that doped and clean sequences can be synthesized on the same oligonucleotide. Whether or not this method is employed, it is an example of how to dissect the chemistry of mixed-site oligonucleotide synthesis. Hermes et al. (1989) have shown that mutations introduced by this method are indeed statistically random.

Regardless of the strategy employed, the level of misincorporation of an oligonucleotide should be decided in advance by the mutagenesis frequency desired. Quantitatively, this level is given by the probability distribution:

$$
P(n, m, x)=[m ! /(m-n) ! n !][x]^{n}[1-x]^{m \times n}
$$


where $P$ is the probability of finding $n$ errors in an oligonucleotide $m$ in length with $x$ level of misincorporation (fraction "wrong" nucleotides delivered). This equation describes a Poisson distribution. If primarily single mutations are desired, then $x$ should be maximized for $n=1$; if multiple mutations (e.g., doubles, or triples in a single oligonucleotide) are necessary, $x$ should be correspondingly higher. If the mix is optimized for $n$ mutations, then $n-1$ and $n+1$ mutations will occur in roughly equal amounts and $n$ mutations will be the most frequent.

Cloning randomized oligonucleotides can be difficult, since a complementary wild-type sequence will generate mismatches that may result in biased correction in vivo. To avoid this problem, second-strand synthesis can be primed from a nonrandom portion of the sequence, or mutually primed synthesis (e.g., CPMB UNIT 8.2) can be utilized. Alternatively, Derbyshire et al. (1986) describe the direct cloning of doped single-strand material with "sticky ends" into a double-stranded cloning vector. Finally, Reidhaar-Olson and Sauer (1988) describe the synthesis of complementary oligonucleotides containing inosine (which can pair with any of the four natural bases) directly across from randomized codons. This method resulted in the successful introduction of a wide variety of mutations into the gene for lambda repressor, although there was a slight compositional bias in cloned sequences.

\section{STRATEGIES FOR OLIGONUCLEOTIDE PURIFICATION}

Deprotected nucleic acids may be purified and isolated by a variety of methods. The method of choice will depend on the availability of resources, the purity required-some methods cannot separate $(n-1)$-mers from $n$-mers-and time considerations. Any of the methods described below can be used to clean up crude material.

\section{Isolation Methods}

\section{Precipitation}

Direct precipitation of the nucleic acids constitutes a fast and efficient purification from contaminating small molecules such as urea and phenol, but does not allow for purification of abortive synthesis products from the full-length one. If oligonucleotide size separation is required, this method should be used in conjunction with some of the other methods described. After deprotection, resuspend the whitish pellet obtained in water. $\mathrm{Add}_{\mathrm{MgCl}}$ to a final concentration of $10 \mathrm{mM}$ and mix along with 5 vol ethanol. Precipitation should be immediate. Freeze the sample briefly at $-20^{\circ}$ or $-70^{\circ} \mathrm{C}$. Centrifuge the precipitated material, wash with $95 \%$ ethanol, dry, and resuspend in water.

Precipitated deoxyoligonucleotides can be used for sequencing or cloning. They can also be used in PCR reactions, although the efficiency of amplification may be reduced as compared to gel-purified oligonucleotides. If the DNA is to be phosphorylated, a more thorough purification procedure is necessary, since $\mathrm{T} 4$ polynucleotide kinase is inhibited by lingering ammonium ions.

\section{Sizing columns}

Size-exclusion chromatography is extremely useful as a final purification step, especially when small-molecule contamination occurs with otherwise pure oligonucleotides, but (like precipitation) does not effect purification of abortive synthesis products from the full-length one. If oligonucleotide size separation is required, this method should be used in conjunction with some of the other methods described. Oligonucleotides purified by PAGE might contain small amounts of low-molecular-weight contaminants such as urea or phenol that might inhibit enzymatic reactions; sizing columns are a simple way to 
decontaminate these samples. Spin columns are simple to prepare and use, but gravityflow columns give better, more reproducible separation. The type of resin used should be adjusted based on the size of the oligonucleotide being purified (e.g., Sephadex G-25 for 25-mers or less, G-50 for longer sequences).

\section{Reversed-phase cartridges}

A hydrophobic matrix may be used to separate full-length from abortive synthesis products if the final trityl group is left on following the final monomer coupling reaction. The resulting hydrophobically tagged full-length "trityl-on" oligonucleotide can be separated easily from failure sequences, which lack trityl groups and do not efficiently bind the hydrophobic matrix. Several companies supply columns designed specifically for the purification of "trityl-on" oligonucleotides (e.g., Applied Biosystems oligonucleotide purification cartridges). The procedure is simple and can be performed on a number of samples in parallel within only a few minutes. The yield from such columns is excellent, often $>80 \%$ of the applied sample. However, although a majority of failure sequences are removed using this method, many shorter sequences are co-purified with the desired full-length material. Some of these fragments are due to cleavage of depurinated DNA. These apurinic molecules can be eliminated prior to cleavage from the column by treatment with lysine (Horn and Urdea, 1988). In addition, if care is not taken to wash and elute samples from these columns slowly, some inhibitors (particularly of ligation reactions) may co-elute.

\section{Denaturing PAGE}

Denaturing polyacrylamide gel electrophoresis (APPENDIX $3 B$ ) separates oligonucleotides with single-residue resolution and is the method of choice for purifying full-length oligonucleotides. However, the compatibility of the chemistries of modified nucleotides incorporated into the nucleic acids and acrylamide matrix should be checked (thiolated oligonucleotides seem to undergo Michael addition to the acrylamide, which renders them irreversibly capped).

\section{HPLC}

Liquid chromatography can also separate oligonucleotides with single-residue resolution, but its chief advantage is speed. Total run time can be as short as $30 \mathrm{~min}$. The use of alkali perchlorate salts has made ion exchange the HPLC method of choice given that long oligonucleotide ( $>40$ residues) may be easily purified in large scales $(\geq 25 \mu \mathrm{mol}$; Sproat et al., 1995; Warren and Vella, 1994). However, secondary structural migration anomalies are generally more severe than those found with PAGE. Depending on the system employed, the amount of oligonucleotide that can be purified in a single chromatographic run can be comparable to PAGE, and sample recovery is typically $>70 \%$. For laboratories with an HPLC system and a need to routinely purify short oligonucleotides with no secondary structure, this method is ideal.

Oligonucleotides can be purified with HPLC by charge differences through ion exchange or hydrophobicity if the final trityl group is left on following the ultimate monomer coupling reaction. Only the "trityl-on" systems use buffers that can be lyophilized. A more complete treatment of the complexities of oligonucleotide purification by HPLC can be found in Applied Biosystems User Bulletin no. 13 on oligonucleotide purification (Applied Biosystems, 1988).

\section{Confirming the Oligonucleotide Sequence}

Most oligonucleotides that are used for cloning need not be checked immediately after synthesis, since the clones themselves will be checked after biological or enzymatic amplification. However, in cases where a sequence will be used in a structural application 
such as mobility-shift assays (e.g., CPMB UNIT 12.1), filter-binding assays (e.g., CPMB UNIT 12.8), or crystallography, it is desirable to confirm the sequence. For almost all oligonucleotides, this usually requires chemical sequencing (e.g., CPMB UNIT 7.5). Although sequencing will confirm that the correct product was made, it cannot determine the homogeneity. How unnatural or protected bases will react during chemical sequencing, or how they will affect mobility on a sequencing gel, is not predictable. In order to determine what fraction of molecules contain only the natural bases (A, T, C, and G), it is necessary to digest the DNA enzymatically to completion and to examine its composition by a comparison with standard bases separated by HPLC. At least one HPLC buffer system has been developed specifically for examining modified nucleosides in chemically synthesized oligonucleotides (Eadie et al., 1987). The insertion and deletion rates for chemical nucleic acid synthesis are non-negligible. The rate of insertions (presumably due to DMT cleavage via tetrazole) has been measured to be as high as $0.4 \%$ per position, and the rate of deletions (presumably due to incomplete capping) has been found to be as high as $0.5 \%$ per position (A. Keefe and D. Wilson, pers. comm.). Therefore, for an oligonucleotide 100 bases in length and assuming a random, noncorrelated mechanism of action, only about $40 \%$ of the sequences will be the intended one. Finally, matrix-assisted laser desorption/ionization mass spectrometry (MALDI-MS; UNIT 10.1), which has recently emerged as a new biotechnology tool, may be used to determine the sequence of deoxy and ribo-oligonucleotides of up to 60 bases (Zhu et al., 1997).

\section{MONITORING DNA SYNTHESIS USING THE TRITYL ASSAY}

A trityl cation is released from the $5^{\prime}$ end of the growing oligonucleotide during each synthesis cycle, and the yield of each step of the synthesis can be determined by spectrophotometrically measuring the amount of trityl cation liberated. This procedure is the simplest and most rapid means available for the identification of problems with synthesized oligonucleotides prior to deprotection and purification. Also, in-line quantitative trityl monitors can be interfaced to most synthesizers (Ana-Gen Technologies).

\section{Materials}

0.1 M para-toluene sulfonic acid (TSA; monohydrate) in acetonitrile (see recipe for dry acetonitrile)

15-ml glass tubes (graduated, if possible)

1. Collect the trityl cation solution in 15-ml glass tubes after each step.

It is helpful to use graduated tubes so that a uniform final volume of acetonitrile can be attained before assay.

2. Dilute the first three and last three fractions to $10 \mathrm{ml}$ with $0.1 \mathrm{M}$ TSA in acetonitrile. Mix thoroughly.

CAUTION: Handle the solution consisting of dichloroacetic acid (or trichloroacetic acid) in dichloromethane and acetonitrile with gloves, because it is corrosive as well as toxic. Avoid prolonged contact with toxic acetonitrile fumes. Do not pipet by mouth.

Although $2.5 \mathrm{ml}$ of deblocking solution is initially released, because acetonitrile is a volatile liquid the volume may change during the course of a synthesis. Fractions may sit for several days before being assayed without affecting the results. Samples that have evaporated to dryness must be thoroughly redissolved. The acid ensures protonation of the trityl groups, making them more strongly colored. It is misleading merely to visualize the yellow/orange color of the fractions, however, since variable volumes of differing acidity are often released.

3. Dilute each sample 20- to 50-fold in the same solution. Measure the absorbance at $498 \mathrm{~nm}$ versus acetonitrile/TSA. 
These dilutions are necessary because most spectrophotometers cannot accurately measure absorbances $>2$.

4. Calculate the stepwise coupling efficiency and absolute yield for the synthesis as a whole. The stepwise efficiency is given by:

$$
(\text { stepwise efficiency })^{n}=\frac{\text { average absorbance of last three fractions }}{\text { average absorbance of first three fractions }}
$$

where $n$ is the number of trityl nucleotides in the oligonucleotide (equal to the length of the oligonucleotide for trityl-off syntheses).

The absolute yield of product is given by:

$$
\mu \mathrm{mol} \text { DMT }=\frac{(\text { absorbance of last fraction }) \times(\text { dilution factor }) \times(10 \mathrm{ml})}{70 \mathrm{ml} / \mu \mathrm{mol}}
$$

where $70 \mu \mathrm{mol}$ is the extinction coefficient of DMT.

The average stepwise efficiency is useful in determining the relative efficiency of each cycle of the synthesis. The absolute yield is useful for determining how many milligrams of product are present for subsequent purifications, although not all of the product will be of the desired length.

The average absorbance of the first and last few steps is used to avoid discrepancies in individual trityl assays. Clearly, if the synthesis was performed on a 1-mmol column, the absolute yield in the first few fractions should be close to $1 \mathrm{mmol}$. A low absolute yield (absorbance) in the first trityl release, followed by higher absolute yields (absorbances) in the next few fractions, is sometimes observed. This is because some spontaneous detritylation occurs during storage of the columns and the trityls are subsequently rinsed off during the prime lines program. In this case, the average stepwise efficiency should be calculated with fractions 2 through 4 . It should be noted that visual assessment of the trityl fractions cannot begin to detect subtle differences $(<5 \%)$ which may be critical in terms of overall yield.

5. Perform troubleshooting assay (see Support Protocol) if average stepwise efficiency is low.

SUPPORT PROTOCOL

Introduction to the Synthesis and Purification of Oligonucleotides

\section{USING THE TRITYL ASSAY FOR TROUBLESHOOTING}

If the average stepwise efficiency for the oligonucleotide synthesis is low, each fraction should be assayed in order to diagnose the problem (what counts as a "low" yield depends on the length and quantity of oligonucleotide desired). In general, synthetic efficiency should be $>99 \%$ per step, although lower stepwise efficiencies can be tolerated for short oligonucleotides ( $<40$ bases) or where yield is not critical.

Three classes of failures can be detected by trityl assays. A low absolute yield at the first step followed by similarly low absorbances that results in a low overall yield is commonly due to inefficient purging of activator or phosphoramidite lines prior to the synthesis. Such a problem frequently occurs when a synthesizer has not been used for several days. Purge the lines with dry reagents prior to starting the run to avoid inefficient synthesis. Most machines have a priming program precisely for this purpose.

If the stepwise efficiency of each step is low, there is a systematic problem with one of the common reagents, such as the acetonitrile. Often this is due to moisture in one or more of the reagents, and it is more likely if reagents have not been recently replaced. The phosphoramidites should be used until they are almost completely exhausted during a 
series of syntheses, so that fresh chemicals will be diluted as little as possible by older, potentially wet material (see Critical Parameters).

Finally, individual trityl assays are most useful in determining when phosphoramidites have become defective. In this case, drops in stepwise efficiency will only be seen at the coupling steps involving the phosphoramidites in question.

Many problems, such as inefficient oxidation or product depurination, cannot be detected by the trityl cation assay. Therefore, the trityl assay procedure should be used in conjunction with HPLC or gel electrophoresis for product analysis, especially if a homogenous population of oligonucleotides is essential.

\section{DEPROTECTION OF DNA OLIGONUCLEOTIDES}

After synthesis is complete, the DNA may be cleaved from the column and protecting groups removed by treatment with ammonia. Although very extended treatment in base can harm DNA, hours of ammoniolysis are still preferred to ensure complete deprotection, since a homogeneous population of "natural" oligonucleotides at slightly lower yield is better than a mixed population of partially deprotected, "unnatural" molecules.

To cleave the DNA from the support matrix and remove protecting groups completely, the support-bound product must be treated with concentrated ammonia at $55^{\circ}$ to $60^{\circ} \mathrm{C}$ overnight ( $\geq 12 \mathrm{hr}$ ). Even with such extended treatment, deoxyguanosine may not be completely deprotected (Schulhof et al., 1987). Raising the temperature at which oligonucleotides are deprotected has been reported to speed up the process $\left(\geq 5 \mathrm{hr}\right.$ at $\left.70^{\circ} \mathrm{C}\right)$.

Phosphoramidites with more labile protecting groups such as phenoxyacetyl or dimethylformadine masking adenosine and guanosine have recently become commercially available (Table A.3C.1). These allow essentially complete deprotection within 30 to 60 min at $70^{\circ} \mathrm{C}$ (Reddy et al., 1994). Also, by replacing the traditional benzyl protection of cytosine with acetyl and using a 1:1 mixture of aqueous ammonium hydroxide and aqueous methylamine, oligonucleotides synthesized with traditional purine protections may be completely deprotected in $5 \mathrm{~min}$ at $65^{\circ} \mathrm{C}$ (Reddy et al., 1995). Finally, anhydrous ammonia gas-phase deprotection of oligonucleotides has recently been described; this provides a convenient method for parallel deprotection of as many columns will fit in a reactor. Since no water is present, the fully deprotected oligonucleotides remain adsorbed to the column matrix, thereby guaranteeing that no cross-contamination will occur. The oligonucleotides can then be eluted with water and desalted or further purified. Using PAC-protected monomers, the cleavage and deprotection processes can be completed in $\sim 30$ min (Boal et al., 1996).

\section{Materials}

Concentrated $(14.8 \mathrm{~N})$ ammonium hydroxide (see recipe)

Triethylamine

3:1 (v/v) concentrated ammonium hydroxide/ethanol

$n$-Butanol

Screw-cap plastic vial (preferably fitted with rubber $\mathrm{O}$ ring)

Heat block or oven, $55^{\circ}$ to $60^{\circ} \mathrm{C}$

$0.2-\mu \mathrm{m}$ filter

1. In a screw-cap plastic vial, suspend the synthesis support matrix or the already support-cleaved oligonucleotide in $\sim 1.0 \mathrm{ml}$ concentrated ammonium hydroxide for a $1-\mu$ mol synthesis.
BASIC

PROTOCOL 2

Commonly Used

Techniques

A.3C.15 
Depending on the synthesizer or nucleic acid provider, the oligonucleotide may come attached to a support matrix or free in an ammonium hydroxide solution. The volume of ammonium hydroxide in which product is eluted from the synthesizer is variable. The ammonium hydroxide used should not have been diluted by excessive vapor loss.

2. Place the sample in heat block or oven for $\geq 12 \mathrm{hr}$ at $55^{\circ}$ to $60^{\circ} \mathrm{C}$.

Seal vial tightly with Parafilm (if not fitted with a rubber $O$ ring).

3. After cleavage from the support and deprotection are complete, spin the sample briefly in a tabletop centrifuge to pool the ammonia and support that may have collected in the cap. Let the vial cool to room temperature before opening it to avoid sample boil-over.

4. Filter off the support by passing the liquid through an $0.2-\mu \mathrm{m}$ filter and wash the filter with $0.3 \mathrm{ml}$ of $3: 1(\mathrm{v} / \mathrm{v})$ ammonium hydroxide/ethanol.

5. Precipitate the oligonucleotide from the resulting supernatant by adding 10 vol $n$-butanol and vortexing for $15 \mathrm{sec}$. Centrifuge $10 \mathrm{~min}$ at $16,000 \times g, 4^{\circ} \mathrm{C}$.

6. Remove and discard the single aqueous $n$-butanol phase to reveal the white oligonucleotide pellet.

Oligonucleotides 20 residues or shorter with good trityl responses are typically suitable for use directly in DNA sequencing, PCR amplification, and gel-shift analysis. However, if more homogenous material is required, methods such as denaturing PAGE and HPLC may be employed to further purify the full length product.

7. If a yellowish liquid or crusty pellet remains, rather than a white powder, resuspend the pellet in $0.1 \mathrm{ml}$ distilled water and precipitate again with $n$-butanol as described above.

Generally, it is not necessary to add additional salt for precipitation.

Further extraction will aid in removing any residual ammonia or volatile organics. If the yellow color does not disappear, it will ultimately be removed by almost any of the standard purification methods.

8. Lyophilize the sample to dryness in a Speedvac evaporator.

This deprotection is primarily intended for oligonucleotides with the trityl group off. When the trityl group is left on, care must be taken that it is not prematurely hydrolyzed from the DNA by acid conditions. During lyophilization, a drop of triethylamine must be added regularly to the sample in order to maintain its basicity (Applied Biosystems, 1988). Heating of the samples should be avoided.

BASIC PROTOCOL 3

Introduction to the Synthesis and Purification of Oligonucleotides A.3C.16

\section{DEPROTECTION OF RNA OLIGONUCLEOTIDES}

Techniques and recommendations for deprotecting RNA are similar to those for DNA. Ammoniolysis cleaves the RNA from the support and frees the bases of their protecting groups. Additionally, the $2^{\prime}$ hydroxyl protecting group must be removed to reveal a functional RNA sequence. Reagents, water, and plasticware to which RNA is exposed must be sterile. The protocol detailed below is for use with nucleosides bearing the standard isobutyryl and benzyl protection on the bases and 2 ' silyl protection. Protection options are becoming available that both increase yield and streamline deprotection time (Table A.3C.1).

\section{Materials}

$100 \%$ ethanol

$3: 1(\mathrm{v} / \mathrm{v})$ concentrated $(14.8 \mathrm{~N})$ ammonium hydroxide/ethanol

$3 \mathrm{M}$ sodium acetate, $\mathrm{pH} 5.2$ (APPENDIX 2A)

Triethylamine trihydrofluoride

Screw-cap plastic vial (preferably fitted with rubber O ring) 
Heat block or oven, $55^{\circ}$ to $60^{\circ} \mathrm{C}$

$0.2-\mu \mathrm{m}$ filter

Sephadex G-25 column (Amersham Pharmacia Biotech)

1. In a screw-cap plastic vial, suspend the synthesis support matrix or the already support-cleaved oligonucleotide in $\sim 1.2 \mathrm{ml}$ of $3: 1(\mathrm{v} / \mathrm{v})$ ammonium hydroxide/ethanol for up to a $1-\mu \mathrm{mol}$ scale.

Depending on the synthesizer or nucleic acid provider, the oligo may come attached to a support matrix or free in an ammonium hydroxide solution. The volume of ammonium hydroxide in which product is eluted from the synthesizer is variable. The ammonium hydroxide used should not have been diluted by excessive vapor loss.

2. Place the sample in a heat block or oven for 12 to $16 \mathrm{hr}$ at $55^{\circ}$ to $60^{\circ} \mathrm{C}$.

Seal vial tightly with Parafilm (if not fitted with a rubber $O$ ring). If fast-cleaving phosphoramidites such as PAC-protected purines and acyl-protected cytosine are used, the deprotection time of the bases can be a little as 10 min in methylamine at $65^{\circ} \mathrm{C}$ (Wincott et al., 1995).

3. After cleavage from the support and base deprotection are complete, spin the sample briefly in a tabletop centrifuge to pool the ammonia and support that may have collected in the cap. Let the vial cool to room temperature before opening it to avoid sample boil-over.

4. Filter off the support by passing the liquid through an $0.2-\mu \mathrm{m}$ filter and wash the filter with $0.3 \mathrm{ml}$ of $3: 1(\mathrm{v} / \mathrm{v})$ ammonium hydroxide/ethanol.

5. Evaporate the combined solutions to dryness in the Speedvac evaporator without heating. Resuspend the pellet in $0.2 \mathrm{ml}$ of $100 \%$ ethanol and evaporate to dryness without heating.

6. Treat the dried residue with triethylamine trihydrofluoride $(0.3 \mathrm{ml}$ for a $0.2-\mu \mathrm{mol}$ or $0.5 \mathrm{ml}$ for a $1-\mu \mathrm{mol}$ synthesis). Allow to rotate in a foil-covered screw-cap vial in the dark for at least 24 but no more than $48 \mathrm{hr}$.

Alternative methods exist to remove the 2' silyl protecting groups either under dilute acidic conditions (Kawahara et al., 1996) or with anhydrous triethylamine/hydrogen flouride in $N$-methylpyrrolidinone (Wincott et al., 1995).

7. To desalt via ethanol precipitation, add an equal volume of water to the triethylamine trihydrofluoride solution and immediately dilute with $1 / 10$ vol of $3.0 \mathrm{M}$ sodium acetate, $\mathrm{pH}$ 5.2. Precipitate by adding $3 \mathrm{vol}$ of $100 \%$ ethanol and chilling for $\sim 20 \mathrm{~min}$ at $-80^{\circ} \mathrm{C}$. Centrifuge $10 \mathrm{~min}$ at $16,000 \times g, 4^{\circ} \mathrm{C}$.

An alternative method exists for isolation of the RNA by diethyl ether precipitation (Song and Jones, 1999).

8. Remove and discard the single ethanol layer to reveal the white oligonucleotide pellet.

9. If a yellowish liquid or crusty pellet remains, rather than a white powder, resuspend the pellet in $0.1 \mathrm{ml}$ distilled water and repeat the sodium acetate/ethanol precipitation as described in step 7. If desired, desalt the RNA on a desalting matrix such as Sephadex G-25.

\section{REAGENTS AND SOLUTIONS}

Use deionized, distilled water in all recipes and protocol steps. For common stock solutions, see APPENDIX 2A; for suppliers, see SUPPLIERS APPENDIX. 
Since large volumes are used for rinsing lines and dissolving other reagents, acetonitrile is one of the most costly reagents in nucleic acid synthesis. If the DNA synthesizer is used infrequently, it may be useful to produce small amounts of dry acetonitrile immediately prior to synthesis since the acetonitrile will accumulate water after the bottle has been opened. Moisture greatly diminishes synthetic yield. In this case acetonitrile can be prepared in any laboratory equipped for routine distillations. However, the time and effort involved in setting up and maintaining a still should be balanced against the cost of obtaining dry acetonitrile. The use of molecular sieving pouches that do not release metal ions is recommend; they are often available from the synthesizer manufacturer and should keep the acetonitrile to $<20 \mathrm{ppm}$ water. Some commercial suppliers now market bulk solvents specifically for nucleic acid synthesis (e.g., Baker "low-water" acetonitrile, $0.002 \%$ or 20 ppm water; Burdick and Jackson acetonitrile, $0.001 \%$ or $10 \mathrm{ppm}$ water). The cost of these special dry reagents is about the same as that of HPLC-grade acetonitrile (which typically contains $0.01 \%$ or $100 \mathrm{ppm}$ water), which is the starting material for a laboratory distillation.

CAUTION: Acetonitrile vapor is poisonous.

\section{Ammonium hydroxide}

Purchase concentrated $(14.8 \mathrm{~N})$ ammonium hydroxide in 0.5 - to 1.0-liter bottles, and store it at $4{ }^{\circ} \mathrm{C}$ to reduce the ammonia gas found in the vapor phase above the liquid.

CAUTION: Concentrated ammonium hydroxide is extremely caustic. Breathing the vapors is harmful. Always use this compound in the fume hood, as it is possible to be quickly overcome by ammonia fumes and blinded.

Ammonia is volatile and its concentration will decrease on repeated opening, until it is no longer completely functional as a deprotecting reagent. When this occurs, obtain a fresh supply.

\section{Oxidizer}

Prepare $0.02 \mathrm{M}$ iodine in 7:2:1 (v/v/v) THF/pyridine/water. Store $\leq 6$ months at room temperature.

CAUTION: Pyridine is toxic in both liquid and vapor forms.

CAUTION: Iodine is harmful if inhaled. Beware of spills containing both iodine and ammonia since explosive compounds can be formed.

Most commercial sources of THF contain BHT, a free-radical scavenger that prevents the buildup of explosive peroxides. This compound has no effect on synthesis chemistry. Any commercial grade of pyridine is acceptable; use resublimed iodine.

\section{Phosphoramidites}

Prepare phosphoramidites in extremely dry, commercially sealed acetonitrile (see recipe) according to the procedure recommended by the synthesizer manufacturer. Store $\leq 6$ months at $4{ }^{\circ} \mathrm{C}$ and discard after 2 weeks.

Some companies recommend dissolving phosphoramidites to give equal molarities of the four bases, while others recommend a standard weight/volume ratio that will give slightly different final molar concentrations. The actual phosphoramidite concentration may matter for some applications-e.g., when making mixed-site oligonucleotides.

Introduction to the Synthesis and Purification of Oligonucleotides

\section{COMMENTARY}

\section{Critical Parameters}

By optimizing the reagents and protocols used in oligonucleotide synthesis, it is reliably possible to make products of greater length and yield, while minimizing the costs associated with unproductive runs. 


\section{Excluding water from solvents}

The most critical factor in any synthesis is how the reagents are handled to exclude water from the system. From the moment a bottle is opened, it is in contact with water in the air, and all the solvents used are hygroscopic and will absorb water vapor, which reduces yields. This problem is so severe that it is advisable to avoid large-scale, lengthy, or important runs on rainy or highly humid days.

Special anhydrous reagents can be purchased from most manufacturers of nucleic acid synthesizers. Additionally, chemical suppliers are now beginning to market solvents specifically for DNA synthesis. Adding molecular sieving pouches to the activator and acetonitrile used before oxidation is extremely useful in preserving the anhydrous environment of the phosphoramidite coupling reaction.

Phosphoramidites are the most sensitive to water contamination because they are easily hydrolyzed, which renders them unreactive. Precautions must be taken to avoid exposing them to even small amounts of water. Very dry acetonitrile ( $<0.003 \%$ water), kept as a separate stock and sealed under argon, should be used to dissolve the phosphoramidites. The acetonitrile should be introduced through the septum on the amidite bottles via a syringe. Glass syringes can be dried in $300^{\circ} \mathrm{C}$ drying oven, then cooled in a desiccator prior to use. Plastic syringes can be dried in a $45^{\circ} \mathrm{C}$ vacuum oven. Disposable plastic syringes from air-tight sterile casings are dry enough to use with acetonitrile to be dispensed into phosphoramidites with no additional precautions. The syringe should be filled with argon or helium prior to drawing up the acetonitrile, so that wet air is not introduced into the system. On some synthesizers, this can be done directly via the phosphoramidite ports. Otherwise, an argon line should be used.

An argon line is generally a helpful tool for DNA synthesis chemistry. It consists of an argon tank with a regulator connected to a piece of plastic (Tygon) tubing. The tubing is then fitted with either a Pasteur pipet or a syringe and needle. Gas flow can be roughly determined via an in-line "bubbler" (e.g., Aldrich). Argon from a tank is dry enough so that an in-line desiccator is unnecessary. It is necessary to flush the line for several seconds prior to use. Empty bottles can be dried under an argon stream, which will help to exclude condensation from the air. Partially used reagents should be sealed under an argon layer to prevent equilibration with water vapor in the air (the heavier argon will exclude air from the containers).

\section{Choosing synthesis columns}

Automated DNA synthesis generally takes place on a solid support made of controlledpore glass (CPG; see UNITS $3.1 \& 3.2$ ). This is a porous, nonswelling particle, 125 to $177 \mu \mathrm{m}$ in diameter, that is derivatized with a terminal nucleotide attached to a long spacer arm. The accessibility of the growing end of the oligonucleotide chain is influenced by the pore size of the particle. It has been recommended that oligonucleotides up to 50 bases in length should be synthesized on CPG with $\sim 500-\AA$ pores, while longer oligonucleotides should be synthesized on CPG with $\sim 1000$ - or larger pores. Be sure that the column geometry is compatible with the trityl monitor. Also, when packing columns be sure to purge the column first with dry acetonitrile and then with dry argon to ensure that all CPG particles are caught between the filters, since loose particles can damage the synthesizer.

CPG columns are available that contain $\sim 0.2$ to $10.0 \mu \mathrm{mol}$ of linked nucleotides. In general larger loadings are used for larger oligonucleotides since as the size of the oligonucleotide increases, the overall yield decreases. Also, subsequent purification steps invariably involve losses, and the amount of product of correct length must be kept high to ensure that there is enough material. Oligonucleotides $>35$ bases should be synthesized on 1.0- $\mu \mathrm{mol} \mathrm{CPG}$ columns. See Synthesizing Long Oligonucleotides for discussion of choosing a column when synthesizing $\geq 100$-base oligonucleotides.

Alternatively, columns that are more heavily derivatized with the $3^{\prime}$ terminal nucleotide are available as solid supports (e.g., Fractosil, from Merck), and a higher yield of product per column (and per amount of reagent delivered) can be obtained using these columns. However, Fractosil is not recommended for synthesizing oligonucleotides $>20$ bases long.

\section{Synthesis chemistry}

Regardless of reagent and final product purity, there are inherent limitations in the chemistry used for the synthesis of oligonucleotides. Therefore, it is necessary to understand the differences between chemically synthesized and biologically derived DNA.

Failure to produce "natural" DNA can be due to the synthesis chemistry used. Methylation of some bases can occur during deprotection with thiophenol in methyl phosphoramidite-based syntheses. Under standard synthesis conditions, these methylated bases can
Commonly Used 
account for up to $7 \%$ of the nucleotides present, with $N$-3-methyl-dT being the primary modified base (Farrance et al., 1989). However, this problem does not always occur-some such syntheses have been reported to contain $99.9 \%$ $\mathrm{dA}, \mathrm{dG}, \mathrm{dT}$, and dC. In general, this problem can be avoided by increasing the time of the thiophenol deprotection step to between 60 and $90 \mathrm{~min}$.

With both methyl and $\beta$-cyanoethyl chemistries, the glycosidic bond of $\mathrm{N}$-protected purine phosphoramidites is subject to hydrolysis during DMT removal with acid. Such depurination eventually leads to strand cleavage during the ammonia deprotection step. N-protected adenosine is more sensitive to depurination than guanosine, and it is most sensitive when located at the $5^{\prime}$ end of an oligonucleotide chain (Tanaka and Letsinger, 1982).

In order to maintain low levels of depurination, the deblocking step should not be longer than $\sim 1$ min. Additionally, the mildest effective acid (DCA rather than TCA) should be used. Occasionally, however, protected nucleotides are supplied as monomethoxytrityl (MMT) esters, which are 10-fold more resistant to acidic detritylation. Oligonucleotides synthesized with MMT as a protecting group are more susceptible to depurination, since the deblocking step must be correspondingly lengthened to achieve complete detritylation and high yields. In cases where only one base is being added as the MMT compound, it is recommended that this step be performed manually, so that the nucleotides are subjected to only one lengthy acid treatment.

Advances in oligonucleotide synthesis chemistry may mitigate the problems associated with depurination. Recently, nucleotides derivatized with protecting groups that render synthesized material less sensitive to depurination have become commercially available (Schulhof et al., 1987; Pharmacia, 1989).

\section{Reagents}

Most reagents may be purchased from the synthesizer manufacturer or from companies that specialize in reagents for nucleic acid synthesis; however, some labs choose to make their own reagents to reduce costs. Notes on the preparation and storage of certain reagents are provided above. Other reagents should probably be purchased from commercial sources, as the preparation of anhydrous materials is more difficult and expensive than most molecular biology labs can support. An appropriate text should be consulted on the medical dangers of all solutions and reagents used in DNA synthe- sis (see Key References). Most suppliers of materials have a fairly extensive list of products available on-line.

\section{Troubleshooting}

\section{Rescue after reagent depletion}

Most synthesizer problems require a visit from the service engineer. However, syntheses during which reagent or solvent bottles run empty can sometimes be rescued. Certain instruments respond to depleted reagents during a synthesis by continuing the synthesis or stopping. For instruments that stop, it may be possible to recover oligonucleotides, depending on which reservoir was depleted. It is important that the lines be rinsed after an empty reservoir has been detected (some machines perform this step automatically). Otherwise, the oligonucleotide should be resynthesized. Many machines have the capacity to restart in the middle of a cycle, and if not, the cycle can be finished manually. The exact procedure will vary depending on which reagent has been exhausted.

Acetonitrile. This is quite serious. The lines cannot be rinsed of whatever reagent they last contained; thus, determine whether to continue with the synthesis based on the reactivity of the last reagent in the lines.

Deblocking reagent. Refill the reservoir. The machine can continue at the beginning of the cycle that was interrupted, but full-length yield will greatly suffer.

Phosphoramidites. Refill the reservoir and perform manual coupling. In this way, the chains that did elongate will not form $n+1$ products, and those that did not will have a chance to elongate. Programmed synthesis can be resumed with the next cycle.

Capping reagent. Perform a manual capping and complete the cycle manually, at which point programmed synthesis can resume. This ensures that capping is efficient for this cycle and avoids accumulation of $n-1$ sequences.

Oxidizer. Running out of oxidizer is particularly dangerous, because the unstable phosphite linkages remain on the column for long periods. It is best to discard the material; otherwise, perform a manual oxidation and continue with the synthesis.

Ammonium hydroxide. Some material may be left on the column if ammonium hydroxide runs out during the course of synthesis. Refill the bottle and pump more ammonium hydroxide through the column, or remove the column and treat with ammonium hydroxide. 
Thiophenol. Refill the thiophenol reservoir. Restart from the deprotection step. At worst, a small amount of product may have cleaved because of inefficient methyl deprotection.

\section{Routine maintenance}

To avoid other purely mechanical problems with a DNA synthesizer, create a regular maintenance schedule to perform a few minor maintenance tasks. Change the various frits and filters that remove debris before they enter the system; furthermore, remove the deposits of salt and debris as these may affect flow rates and decrease volumes delivered. Change the filters on the acetonitrile bottle more often, because the flow of this reagent is much greater than any other. Also, change any rubber O rings every few months. Rinse all the instrument's lines with base and organic solvents thoroughly every $500 \mathrm{hr}$ of machine use or approximately every 3 months. Finally, rinse the lines extensively with dry acetonitrile. Finish the general maintenance by checking the flow rates of the instrument after cleaning and then synthesize a small oligonucleotide. Monitor the trityls carefully in order to confirm that the cleaning did not affect any aspect of the synthesis cycle. If this regimen is followed, many minor delays encountered in the synthesis schedule can be avoided.

\section{Anticipated Results}

When the synthesizer is working properly ( $>98 \%$ coupling efficiency), typical yields of deoxyoligonucleotides 20 bases in length at the $1-\mu \mathrm{mol}$ scale, as measured from the trityl response, are nearly $60 \%$. Also, these shorter deoxyoligonucelotides are typically suitable for DNA sequencing, PCR amplification, and gel-shift analysis without extensive purification. However, if very homogenous material is required, further purification must be done, because many sequences will be truncated. Longer DNA sequences are generally obtained in lesser yield and quality and should be purified. Sequences of $>120$ bases are generally obtained in $\leq 10 \%$ yield as measured by the trityl response. Yields for RNA sequences are typically much lower than for their DNA counterparts of a given length, and the products must be extensively purified.

\section{Time Considerations}

On a typical day when most of the bottles on the synthesizer need refilling, it may take 30 to $60 \mathrm{~min}$ to fill the bottles, empty waste, install columns, and rinse and prime the lines prior to starting a synthesis. The most time-consuming syntheses are those involving doped oligonucleotides, which require a high level of precision in distributing the various phosphoramidites to the appropriate bottles. A computer interfaced with the instrument can increase synthesis accuracy and throughput. Instruments take varying amounts of time to synthesize oligonucleotides depending on the number of columns in use, the length of the oligonucleotide, and the synthesis program. Deprotection and isolation of a standard DNA oligonucleotide will probably take one working day, while RNA oligonucleotides take longer because of the extra deprotection and desalting steps.

\section{Literature Cited}

Applied Biosystems. 1988. User Bulletin no. 13. Applied Biosystems, Foster City, Calif.

Bartel, D. and Szostak, J.W. 1993. Isolation of new ribozymes from a pool of random sequences. Science 261:1411-1418.

Boal, J.H., Wilk, A., Harindranath, N., Max, E.E., Kempe, T., and Beaucage, S.L. 1996. Cleavage of oligodeoxyribonucleotides from controlledpore glass supports and their rapid deprotection by gaseous amines. Nucl. Acids Res. 24:31153117.

Ciccarelli, R.B., Gunyuzlu, P., Huang, J., Scott, C., and Oakes, F.T. 1991. Construction of synthetic genes using PCR after automated DNA synthesis of their entire top and bottom strands. Nucl. Acids Res. 19:6007-6013.

Cohen, G., Deutsch, J., Fineberg, J., and Levine, A. 1997. Covalent attachment of DNA oligonucleotides to glass. Nucl. Acids Res. 25:911-912.

Derbyshire, K.M., Salvo, J.J., and Grindley, N. 1986. A simple and efficient procedure for saturation mutagenesis using mixed oligodeoxynucleotides. Gene 46:145-152.

Eadie, J.S., McBride, L.J., Efcavitch, J.W., Hoff, L.B., and Cathcart, R. 1987. High-performance liquid chromatographic analysis of oligodeoxyribonucleotide base composition. Anal. Biochem. 165:442-447.

Farrance, I.K., Eadie, J.S., and Ivarie, R. 1989. Improved chemistry for oligodeoxyribonucleotide synthesis substantially improves restriction enzyme cleavage of a synthetic $35 \mathrm{mer}$. Nucl. Acids Res. 17:1231-1245.

Gait, M.J. (ed.). 1984. Oligonucleotide Synthesis: A Practical Approach. IRL Press, Washington, D.C.

Gillam, S. and Smith, M. 1980. Use of E. coli polynucleotide phosphorylase for the synthesis of oligodeoxyribonucleotides of defined sequence. Methods Enzymol. 65:687-701.

Hermes, J.D., Parekh, S.M., Blacklow, S.C., Kuster, H., and Knowles, J.R. 1989. A reliable method for random mutagenesis: The generation of mutant libraries using spiked deoxyribonucleotide primers. Gene 184:143-151.
Commonly Used 
Horn, T. and Urdea, M.S. 1988. Solid supported hydrolysis of apurinic sites in synthetic oligonucleotides for rapid and efficient purification on reverse-phase cartridges. Nucl. Acids Res. 16:11559-11571.

Kawahara, S., Wada. T., and Sekine, M. 1996. Unprecedented mild acid-catalyzed desilyation of the 2-O-tert-butyldimethylsilyl group from chemically synthesized oligoribonucleotides intermediates via neighboring group participation of the internucleotide phosphate residue. J. Am. Chem. Soc. 118:9461-9468.

Kinoshita, Y., Nishigaki K., and Husimi Y. 1997. Fluorescence-, isotope- or biotin-labeling of the 5 '-end of single-stranded DNA/RNA using T4 RNA ligase. Nucl. Acids Res. 25:3747-3748.

Oliphant, R. 1989. Functional Sequences from Random DNA. Harvard University Thesis, Boston, Mass.

Oliphant, R., Nussbaum, A.L., and Struhl, K. 1986. Cloning of random-sequence oligodeoxynucleotides. Gene 44:177-183.

Pharmacia. 1989. Analects Vol. 17, no. 2. Pharmacia Biotech, Piscataway, NJ.

Ratliff, R.L. 1982. Terminal deoxynucleotidyltransferase. In The Enzymes, Vol. XV (P.D. Boyer, ed.) pp. 105-118. Academic Press, San Diego.

Reddy, M.P., Hanna, N.B., and Farooqui, F. 1994. Fast cleavage and deprotection of oligonucleotides. Tetrahedron Lett. 35:4311-4314.

Reddy, M.P., Farooqui, F., Hanna, N.B. 1995. Methylamine deprotection provides increased yield of oligoribonucleotides. Tetrahedron Lett. 36:8929-8932.

Reidhaar-Olson, J.F. and Sauer, R.T. 1988. Combinatorial cassette mutagenesis as a probe of the informational content of protein sequences. Science 241:53-57.

Schulhof, J.C., Molko, D., and Teoule, R. 1987. The final deprotection step in oligonucleotide synthesis is reduced to a mild and rapid ammonia treatment by using labile base-protecting groups. Nucl. Acids Res. 15:397.

Song, Q. and Jones, R.A. 1999. Use of silyl-ethers as fluoride scavengers in RNA synthesis. Tetrahedron Lett. 40:4653-4654.

Sproat, B., Colonna, F., Mullah, B., Tsou, D., Andrus, A., Hampel, A., and Vinayak, R. 1995. An efficient method for the isolation and purification of oligoribonucleotides. Nucleosides Nucleotides 14:255-273.

Szostak, J. 1992. In vitro genetics. Trends Biochem. Sci. 17:89-93.

Tanaka, T. and Letsinger, R.L. 1982. Syringe method for stepwise chemical synthesis of oligonucleotides. Nucl. Acids Res. 10:3249.

Uhlenbeck, O.C. and Gumport, R.I. 1982. T4 RNA ligase. In The Enzymes, Vol. XV (P.D. Boyer, ed.) pp. 31-58. Academic Press, San Diego.

Introduction to the Synthesis and Purification of Oligonucleotides A.3C.22 agren, R.J. 1987. Automated chemical synthesis of long oligoribonucleotides using 2'-O-sily- lated ribonucleotide 3 '- $O$-phosphoramidites on a controlled-pore glass support: Synthesis of a 43 nucleotide sequence similar to the $3^{\prime}$ half molecule of an E. coli formylmethionine tRNA. J. Am. Chem. Soc. 109:7845-7854.

Vargeese, C., Carter, J., Yegge, J., Karivjansky, S., Settle, A., Kropp, E., Peterson, K., and Peiken, W. 1998. Efficient activation of nucleoside phosphoramidites with 4,5-dicyanoimidazole during oligonucleotide synthesis. Nucl. Acids Res. 26:1046-1050.

Warren, W.J. and Vella, G. 1994. Analysis and purification of synthetic oligonucleotides by highperformance liquid chromatography. Methods Mol. Biol. 233-264.

Wincott, F., DiRenzo, A., Shaffer, C., Grimm, S., Tracz, D., Workman, C., Sweedler, D., Gonzalez, C., Scaringe, S., and Usman, N. 1995. Synthesis, deprotection, analysis and purification of RNA and ribozymes. Nucl. Acids Res. 23:2677-2684.

Zhu, Y., He, L., Srinivasan, R., and Lubman, D. 1997. Improved resolution in the detection of oligonucleotides up to 60-mers in matrix-assisted laser desorption/ionization time-of-flight mass spectrometry using pulsed-delayed extraction with a simple high voltage transistor switch. Rapid Commun. Mass Spectrom. 11:987-992.

Zon, G., Gallo, K.A., Samson, C.J., Shao, K., Summers, M.F., and Byrd, R.A. 1985. Analytical studies of "mixed sequence" oligodeoxyribonucleotides synthesized by competitive coupling of either methyl or $\beta$-cyanoethyl- $N, N$-diisopropylamino phosphoramidite reagents, including $2^{\prime}$ deoxyinosine. Nucl. Acids Res. 13:8181-8196.

\section{Key References}

Applied Biosystems, 1988. See above.

A well-organized overview of synthetic oligonucleotide synthesis, purification, and quantitation.

Bretherick, L.,1986. Hazards in the Chemical Laboratory, 4th ed. Alden Press, Oxford.

A guide to hazardous chemical handling.

Gait, 1984. See above.

The seminal text on synthetic oligonucleotide synthesis that provides critical insight.

\section{Internet Resources}

http://www.interactiva.de/

Web site detailing synthesis chemistries, procedures, and reagents for solid-phase oligonucleotide chemistry.

Contributed by Andrew Ellington

University of Texas

Austin, Texas

Jack D. Pollard, Jr.

Harvard Medical School and Massachusetts

General Hospital

Boston, Massachusetts 